\title{
Adaptively Applying Modus Ponens in Conditional Logics of Normality
}

\author{
Christian Straßer \\ Centre for Logic and Philosophy of Science, Ghent University (UGent) \\ Blandijnberg 2, 9000 Gent, Belgium \\ Email: christian.strasser@UGent.be, \\ Phone: ++329264 39 79, Fax: ++3292644187
}

\begin{abstract}
This paper presents an adaptive logic enhancement of conditional logics of normality that allows for defeasible applications of Modus Ponens to conditionals. In addition to the possibilities these logics already offer in terms of reasoning about conditionals, this way they are enriched by the ability to perform default inferencing. The idea is to apply Modus Ponens defeasibly to a conditional $A \rightsquigarrow B$ and a fact $A$ on the condition that it is "safe" to do so concerning the factual and conditional knowledge at hand. It is for instance not safe if the given information describes exceptional circumstances: although birds usually fly, penguins are exceptional to this rule. The two adaptive standard strategies are shown to correspond to different intuitions, a skeptical and a credulous reasoning type, which manifest themselves in the handling of so-called floating conclusions.

RÉSUMÉ. A définir par la commande \resume $\{.$. \}

KEYWORDS: defeasible reasoning, adaptive logic, conditional logics of normality, default inferencing, modus ponens, nonmonotonic logic

MOTS-CLÉS : A définir par la commande \motscles $\{\ldots\}$
\end{abstract}

$1^{\text {re }}$ soumission à Journal of Applied Non-Classical Logics, le November 2, 2011 


\section{Introduction}

\subsection{Some Background}

Since the early eighties, default reasoning, i.e., reasoning on the basis of what is normally or typically the case, has drawn much attention from philosophical logicians as well as scholars working in Artificial Intelligence. This is not surprising concerning the prominent role which reasoning on the basis of notions such as normality and typicality has. It clearly occupies a central place from everyday common sense reasoning to expert reasoning in many domains. Thus, logicians are urged to develop formal models which accurately explicate these reasoning forms.

In recent years the traditional formalisms of default reasoning such as presented in the landmark articles on default logic (Reiter, 1980), on circumscription (McCarthy, 1980), and on autoepistemic logic (Moore, 1984) have been criticized and alternative conditional approaches have been developed.

In pioneering works on logics of conditionals the main interest was to model conditionals in everyday language which have the form "if ... then". Most of the research in this domain has been in the vein of the following influential conditional logics: Stalnaker (Stalnaker, 1968) and Lewis (Lewis, 2000) who offer an ontic interpretation of the conditional, Adams (Adams, 1975) who introduces probabilities in the discussion, and Gärdenfors' belief revision principles which are more concerned with acceptability than probability and truth (Gärdenfors, 1978).

There has been, especially since the late eighties, an increasing interest in making use of techniques and properties of conditional logics within the field of nonmonotonic reasoning, such as employed in default reasoning or reasoning with respect to prima facie obligations. The focus of this paper is on conditional logics of normality that have been inspired by pioneering works such as (Boutilier, 1994a; Lamarre, 1991; Kraus et al., 1990). There, a statement of the form $A \rightsquigarrow B$ is read as "From $A$ normally/typically follows $B$ " or "If $A$ is the case then normally/typically also $B$ is the case". We will call " $A \rightsquigarrow B$ " a conditional, and a sequence of conditionals, written $A_{1} \rightsquigarrow A_{2} \rightsquigarrow \ldots \rightsquigarrow A_{n}$ as an abbreviation for $\left(A_{1} \rightsquigarrow A_{2}\right) \wedge\left(A_{2} \rightsquigarrow A_{3}\right) \wedge$ $\cdots \wedge\left(A_{n-1} \rightsquigarrow A_{n}\right)$, an argument.

Conditional logics are attractive candidates for dealing with default reasoning for various reasons: First, the conditional $\rightsquigarrow$ does not have unwanted properties such as Strengthening the Antecedent, from $A \rightsquigarrow B$ infer $(A \wedge C) \rightsquigarrow B$, Transitivity, from $A \rightsquigarrow B$ and $B \rightsquigarrow C$ infer $A \rightsquigarrow C$, and Contraposition, from $A \rightsquigarrow B$ infer $\neg B \rightsquigarrow \neg A$. That the validity of any of these properties leads to undesired results in the context of reasoning on the basis of normality is well-known. Take, for instance, Strengthening the Antecedent: although birds usually fly, $b \rightsquigarrow f$, penguins do not, $(b \wedge$ $p) \rightsquigarrow \neg f$. Thus $(b \wedge p) \rightsquigarrow f$ should not be derived. To find similar counterexamples for the other properties is left to the reader (see e.g., (Boutilier, 1994a) p. 92.). Another advantage is the naturalness and simplicity of the representation of default knowledge by conditionals $A \rightsquigarrow B$ compared to the cumbersome representation by the classical 
approaches mentioned above. The latter use rules such as $A \wedge \pi(B) \supset B$ where $\pi(B)$ expresses for instance that we do not believe $\neg B$ in the case of autoepistemic logic, or that $B$ can consistently be assumed in the case of default reasoning. Furthermore, certain disadvantages of the classical approach can be avoided in the framework of conditional logics. Boutilier for instance argues that certain paradoxes of material implication are inherited by the classical approaches due to the way default knowledge is represented in them (see (Boutilier, 1994a) pp. 89-90).

Starting from the pioneering works such as (Boutilier, 1994a; Delgrande, 1988; Kraus et al., 1990; Lamarre, 1991) there has been vigorous research activity on conditional logics of normality. To mention a few: they have been applied to belief revision in (Boutilier, 1994b; Wobcke, 1995), strengthenings have been proposed for instance to give a more sophisticated account of Strengthening the Antecedent (see (Lehmann et al., 1992; Pearl, 1990)), a labeled natural deduction system has been introduced in (Broda et al., 2002), and various authors have investigated tableaux methods and sequent calculi for conditional logics (see e.g. (Schröder et al., 2010; Giordano et al., 2006; Schröder et al., 2010)). Furthermore, the influential work in (Kraus et $a l ., 1990$ ) is greatly generalized in (Arieli et al., 2000) by their plausible nonmonotonic consequence relations, and in (Friedman et al., 1996) by their plausibility measures.

There is a remarkable agreement concerning fundamental properties for default reasoning in the various formal models. These properties have been dubbed conservative core by Pearl and Geffner (Geffner et al., 1992) and are also commonly known as the KLM-properties (see (Kraus et al., 1990)). Some of the most interesting and important problems in this field are, on the one hand, related to a proper treatment of irrelevant information (see (Delgrande, 1988)) and, on the other hand, to a proper treatment of specificity.

\subsection{Contribution and Structure of this Paper}

This paper tackles another important problem related to conditional logics of normality: while they are able to derive from conditional knowledge bases, i.e., sets of conditionals, other conditionals, their treatment of factual knowledge is mostly rather rudimentary. This concerns most importantly their treatment of Modus Ponens (MP), i.e., to derive $B$ from $A$ and $A \rightsquigarrow B$. We will also speak about detaching $B$ from $A \rightsquigarrow B$ in case $A$ is valid. Usually we do not only have a conditional knowledge base at hand but also factual information $\mathcal{F}$. In order to make use of the knowledge base, it is in our primary interest to derive, given $\mathcal{F}$, what normally should be the case. It goes without saying that for the practical usage of a conditional knowledge base this kind of application to factual information is essential and that the proper treatment of MP for conditionals is a central key to its modeling.

It is clear that full MP should not be applied unrestrictedly to conditional assertions: although birds usually fly, $b \rightsquigarrow f$, we should not deduce that a given bird flies if we also know that it is a penguin, since penguins usually do not fly, $p \rightsquigarrow \neg f$. How- 
ever, if we do not know anything about it than the fact that it is a bird, MP should be applied to $b \rightsquigarrow f$ and $b$. Furthermore, it would be useful if this application is of a defeasible kind, since later we might learn that the bird in question is after all a penguin or a kiwi.

In this paper a simple generic method is presented to enrich a given conditional logic of normality $\mathbf{L}$ by a defeasible MP. We consider $\mathbf{L}$ to consist at least of the core properties (see Section 2). We will refer to $\mathbf{L}$ as the base logic. As hinted above, there are several circumstances when we do not want to apply MP: cases of specificity such as the example with the penguin, or cases in which conditionals conflict, such as the well-known Nixon-Diamond. The central idea presented in this paper is to apply MP conditionally, namely on the condition that it is safe to apply it. This idea will informally be motivated and outlined in Section 3. Formally, the conditional applications of MP are realized by adaptive logics, namely $\mathbf{D L p} \mathbf{p}^{\mathbf{m}}$ and $\mathbf{D L p} \mathbf{p}^{\mathbf{r}}$ (see Definition 5, page 14). The idea of adaptive logics is to interpret a premise set "as normally as possible" with respect to a certain standard of normality. They allow for some rules to be applied conditionally. I introduce adaptive logics formally in Section 4. In our case, as demonstrated in Section 5, MP is going to be applied as much as possible, i.e., as long as no cases of overriding via specificity or similar conflicts take place concerning the conditionals to which MP is going to be applied. That is to say, we are going to apply MP to $A \rightsquigarrow B$ and $A$ on the condition that the other factual information at hand does not describe exceptional circumstances with respect to $A$. As a consequence, detachment from $b \rightsquigarrow f$ and $b$ is for instance blocked if $p$ is the case.

It will be demonstrated that choosing different adaptive strategies serves different intuitions: one corresponding to a more skeptical and the other one corresponding to a more credulous type of reasoning. This difference manifests itself in the handling of so-called floating conclusions. ${ }^{1}$

I will spend some time in demonstrating the modus operandi of the proposed logics and thereby their strengths by having a look at various benchmark examples. In Section 6 I highlight some advantages of the adaptive logic approach, compare it to other approaches, and discuss some other related issues. The semantics are investigated in the Appendix.

\section{Conditional Logics, Their Core Properties and Related Work}

Conditional logics are often presented in terms of extending classical propositional logic with a conditional operator $\rightsquigarrow .{ }^{2}$ Our language is defined by the $(\wedge, \vee, \supset, \neg, \equiv)$ -

1. A floating conclusion is a proposition that can be reached by two conflicting and equally strong arguments.

2 . In some conditional logics of normality $\rightsquigarrow$ is not primitive. For instance in (Boutilier, 1994a) it is defined by making use of modal logic. There the core properties are shown to be equivalent to an extension of the modal logic S4. See (Friedman et al., 1996) for a comparative 
closure of the set of propositional variables and conditionals of the form $A \rightsquigarrow B$, where $A$ and $B$ are classical propositional formulas. Hence, to keep things simple we do not consider here nested occurrences of $\rightsquigarrow$ and focus on flat conditional logics. We refer to $A$ as the antecedent and to $B$ as the conclusion of the conditional. We write $\mathcal{W}$ for the set of all classical propositional formulas (i.e., formulas without occurrences of $\rightsquigarrow)$. We abbreviate $(A \rightsquigarrow B) \wedge(B \rightsquigarrow A)$ by $A \sim B$ and $\neg(A \rightsquigarrow B)$ by $A \not \rightarrow$ $B$. Furthermore, we require that a conditional logic $\mathbf{L}$ satisfies the following core properties, where CL is classical propositional logic (see (Kraus et al., 1990)): ${ }^{3}$

$$
\begin{array}{r}
\text { If } \vdash_{\mathrm{CL}} A \equiv B, \text { then } \vdash(A \rightsquigarrow C) \equiv(B \rightsquigarrow C) \\
\text { If } \vdash_{\mathrm{CL}} B \supset C, \text { then } \vdash(A \rightsquigarrow B) \supset(A \rightsquigarrow C) \\
\\
\vdash A \rightsquigarrow A \\
\vdash((A \rightsquigarrow B) \wedge((A \wedge B) \rightsquigarrow C)) \supset(A \rightsquigarrow C) \\
\vdash((A \rightsquigarrow B) \wedge(A \rightsquigarrow C)) \supset((A \wedge B) \rightsquigarrow C) \\
\vdash((A \rightsquigarrow C) \wedge(B \rightsquigarrow C)) \supset((A \vee B) \rightsquigarrow C)
\end{array}
$$

[RCEA]

The logic defined by these rules and axioms is $\mathbf{P}$. Note that for instance the following properties are valid in $\mathbf{P}{ }^{4}$

$$
\begin{array}{r}
\vdash((A \rightsquigarrow B) \wedge(A \rightsquigarrow C)) \supset(A \rightsquigarrow(B \wedge C)) \\
\vdash((A \wedge B) \rightsquigarrow C) \supset(A \rightsquigarrow(B \supset C)) \\
\vdash((A \sim B) \wedge(B \rightsquigarrow C)) \supset(A \rightsquigarrow C) \\
\vdash_{\mathrm{CL}} A \supset B, \text { then } \vdash A \rightsquigarrow B
\end{array}
$$

We consider these properties to be valid for all the conditional logics of normality in the remainder. Adding the following Rational Monotonicity principle to the core properties yields logic $\mathbf{R}$ (see (Lehmann et al., 1992)): ${ }^{5}$

$$
\vdash((A \rightsquigarrow C) \wedge(A \not \neg \neg B)) \supset((A \wedge B) \rightsquigarrow C)
$$

study of various semantic systems for the core properties such as the preferential structures of (Kraus et al., 1990), the $\epsilon$-semantics of (Pearl, 1989), the possibilistic structures of (Dubois et al., 1991) and $\kappa$-rankings of (Goldszmidt et al., 1992; Spohn, 1988).

3 . We will use the name convention that is associated with conditional logics of normality (see (Chellas, 1975), (Nute, 1980)) and not the one associated with nonmonotonic consequence relations which is used e.g. in (Kraus et al., 1990).

4. The proofs are fairly standard and can be found e.g. in (Kraus et al., 1990).

5. I adopt the names $\mathbf{P}$ and $\mathbf{R}$ for these logics from (Giordano et al., 2006). Although these are the same names as used for the systems in the pioneering KLM paper (Kraus et al., 1990), the reader may be warned: the approach in terms of conditional logics differs from the KLM perspective which deals with rules of inference rather than with axioms. Also, strictly speaking, Rational Monotonicity as defined in (Kraus et al., 1990) is a rule of inference whereas (RM) as defined above is an axiomatic counterpart to it. 
The core properties are not without criticism. On the one hand, it has been pointed out that certain principles of $\mathbf{P}$ resp. $\mathbf{R}$ are not always perfectly intuitive. For instance, Neufeld (Neufeld, 1989) has argued against [CA], Poole (Poole, 1991) against [CC], and Stalnaker (Stalnaker, 1994) and Giordano et al. (Giordano et al., 2010b; Giordano et al., 2010a) against [RM]. ${ }^{6}$

On the other hand, the core properties have been criticized for being too weak. Many nonmonotonic strengthenings have been developed in order to overcome certain weaknesses.

Rational closure (see e.g. (Lehmann et al., 1992; Goldszmidt et al., 1990; Freund, 1997)) for instance strengthens $\mathbf{R}$ by means of a Shoham-like preferential semantics (Shoham, 1987; Shoham, 1988). The idea is to assign natural numbers, i.e. ranks, to formulas. The rank indicates how exceptional a formula is. If for instance $(A \vee$ $\neg A) \rightsquigarrow A$ then $A$ has the lowest rank, 0 . In our penguin example $p$ is of a higher rank than $b$ since after all $(p \vee b) \rightsquigarrow \neg b$. Each formula is ranked as low as possible. A default $A \rightsquigarrow B$ is in the rational closure of a set of defaults $D$ iff the rank of $A$ is strictly less than the rank of $A \wedge \neg B$. In this way a significant problem of $\mathbf{P}$ and $\mathbf{R}$ is tackled, namely its suboptimal treatment of irrelevant information. For instance, the proposition "Tweety is a green bird.", $g$, will get the same rank as "Tweety is a bird.", $b$. Hence, the Rational Closure of $\{b \rightsquigarrow f, g \rightsquigarrow b\}$ contains the default $(b \wedge g) \rightsquigarrow f$, that green birds fly. The latter is neither entailed by $\mathbf{P}$ nor by $\mathbf{R}$. Rational Closure has been shown to be equivalent to Pearl's system Z (see (Pearl, 1990; Goldszmidt et al., 1990)) which employs a probabilistic interpretation of defaults. These and similar approaches have been criticized for inheriting some of the weaknesses of the core properties (see e.g. (Geffner et al., 1992)) resp. of rational monotonicity (see (Giordano et al., 2010a)) and for introducing new problems (see Example 8).

Giordano et al. introduce another preferential semantics based on $\mathbf{P}$ and a tableaux calculus for it. Their system $\mathbf{P}_{\text {min }}$ selects models that minimize non-typical worlds with respect to a given set of formulas. Adding to our example the conditional $p \rightsquigarrow a$, that penguins live in the arctic, $\mathbf{P}_{\min }$ concludes nonmonotonically that there are no penguins that do not live in the arctic: $(p \wedge \neg a) \rightsquigarrow \perp$. This is not a consequence of $\mathbf{Z}$ resp. Rational Closure. However, $\mathbf{P}_{\min }$ 's treatment of irrelevant information is suboptimal: unlike $\mathbf{Z}$ and Rational Closure $\mathbf{P}_{\min }$ does not lead to the consequence $(b \wedge g) \rightsquigarrow f$.

Lehmann's Lexicographic Closure (see (Lehmann, 1995; Benferhat et al., 1993)) improves on some of the shortcomings of Rational Closure by strengthening it further. ${ }^{7}$ On the one hand, it introduces a more rigorous approach to strengthening the

6. Some weakening or variants of Rational Monotonicity have been proposed: e.g. $\vdash((A \rightsquigarrow$ $B) \wedge((A \wedge C) \not \leftrightarrow \neg B)) \supset((A \wedge C) \rightsquigarrow B)$ (IRR) in the context of Description Logic by Giordano et al. (Giordano et al., 2009) or in the context of conditional deontic logics $\vdash((A \rightsquigarrow$ $B) \wedge(A \not \nrightarrow \neg(B \wedge C))) \supset((A \wedge C) \rightsquigarrow B)$ (WRM) by Goble in (Goble, 2004).

7. More precisely, Lexicographic Closure strengthens Rational Closure for all defaults with antecedents that have a finite rank: if $A$ has finite rank and $A \rightsquigarrow B$ is in the rational closure of $D$, then $A \rightsquigarrow B$ is in the lexicographic closure of $D$. 
antecedent and hence avoids the so-called Drowning Problem (we discuss this in more detail in Section 6). On the other hand, it makes sure that in cases of contradictory defaults quantitatively as many defaults as possible are satisfied. The policy is to strictly prefer more specific defaults over less specific ones. The quantitative aspect makes the Lexicographic Closure dependent on the way defaults are presented.

The maximum entropy approach of (Goldzsmidt et al., 1993) is in the probabilistic tradition of the 1-entailment of system $\mathbf{Z}$. It follows a similar intuition as Lexicographic Closure concerning conflicting defaults. One difference is, however, that in some cases the violation of a more specific default may lead to a higher overall entropy than the violation of some less specific defaults and may be thus preferred.

In his critical discussion of the core properties Delgrande (Delgrande, 2006) points out that there are two interpretations of conditionals $A \rightsquigarrow B$. Many approaches, such as the ones listed above, treat defaults as weak material implications that have a defeasible character, e.g. in specificity cases. He identifies several counter-intuitive instances where the core properties obtain contrapositives of defaults. This, so he argues, is a result of treating default conditionals in terms of material implications rather than in terms of inference rules. In the spirit of the latter perspective he develops a system based on a weakened core logic (in comparison to $\mathbf{P}$ ). He demonstrates that his rule-based system has a lot of nice properties in terms of treating irrelevant information and conflicting defaults. Another rule-based approach is e.g. presented by Dung and Son in (Dung et al., 2001).

The take on defaults in terms of weak material implications is very obvious in approaches that make abnormality assumptions explicit (see McCarthy's Circumscription (McCarthy, 1980), Geffner and Pearl's Conditional Entailment (Geffner et al., 1992), as well as the one presented in this paper). Here a default $A \rightsquigarrow B$ is presented by $A \wedge \alpha \supset B$ (or by both in the case of Conditional Entailment) where $\alpha$ expresses normality conditions that have to hold for this default. The interesting aspect of conditional entailment is that it extracts a priority order on the normality assumptions automatically from the knowledge base. The idea is to interpret a given knowledge base such that the normality assumptions of the defaults are validated "as much as possible". The priority order takes care that in case of conflicts more specific defaults are preferred where possible.

We conclude this section by noting that conditional logics have been successfully applied to various fields. For instance their relevance for belief revision has been investigated in (Wobcke, 1995; Boutilier, 1994b; Booth, 2001). The description logic $\mathcal{A L C}$ has been enhanced with a "typicality" operator in (Giordano et al., 2009). Similar to the logics that are going to be presented in the present paper this system allows for inferences on the basis of factual information. However, in its current form the logic faces the problem of irrelevance pointed out above: given the information that typical birds fly the logic does not allow to infer that typical green birds fly. In order to deal with such problems the authors propose to integrate "a standard mechanism to reason about defaults" (p. 14) which is left for future research. Furthermore, recently conditional logics have been applied to access control and security in (Genovese et 
al., 2011). There the authors extend Garg and Abadi's access control logic ICL from (Garg et al., 2008) with intuitionistic conditional logic.

\section{Modus Ponens in Conditional Logics of Normality}

In this section I will informally motivate and outline the main idea behind the modeling of a defeasible MP in this paper.

A naïve way to apply MP would be to use the unrestricted version

$$
\vdash((A \rightsquigarrow B) \wedge A) \supset B \quad \text { [MP } \rightsquigarrow]
$$

However, this would lead to logical explosion whenever we are confronted with conflicting defaults, for instance in cases of specificity. Informally speaking, specificity occurs if a more specific argument overrides a more general one. One way to formalize this is as follows: if $A$ is the case and $A \rightsquigarrow B \rightsquigarrow C$, as well as $A \rightsquigarrow \neg C$, then $B \rightsquigarrow C$ is overridden by $A \rightsquigarrow \neg C$, or in terms of arguments, $A \rightsquigarrow B \rightsquigarrow C$ is overridden by $A \rightsquigarrow \neg C$. The reader finds an illustration in Figure 1a. The illustrations in Figures 1 and 2 have to be read in a similar way as inheritance networks (see (Horty, 1994)): nodes are in our case propositions, " $A \rightarrow B$ " indicates $A \rightsquigarrow B$, " $A \cdots B$ " indicates $A \rightsquigarrow \neg B$, “ $A \Rightarrow B$ " indicates $A \vdash_{\mathbf{C L}} B$, and " $A=\Rightarrow B$ " indicates $A \vdash_{\mathbf{C L}} \neg B$.

Example 1. A standard example illustrating a case of specificity is the following (see Figure 1b):

- Birds normally fly. $-b \rightsquigarrow f$

- Penguins are (normally) birds. $-p \rightsquigarrow b$

- Penguins normally do not fly. $-p \rightsquigarrow \neg f$

The information represented by $p$ is less specific or normal than the information represented by $b$. Thus, obviously the more specific $p \rightsquigarrow \neg f$ overrides $b \rightsquigarrow f$. This has an important consequence: Given $p \wedge b$ or $p$ we do not want to apply MP to $b$ and $b \rightsquigarrow f$. However, if we only have $b$ as factual knowledge it would be justified on the basis of default reasoning to apply MP to $b$ and $b \rightsquigarrow f$.

Since, as argued above, full MP is highly problematic in the context of default inferencing, we will in the remainder make use of a restricted MP. The idea is to restrict MP to "safe" antecedents. In order to express this, we introduce a unary operator • into our language which is applicable to propositional formulas. $\bullet A$ expresses that the given factual information is atypical or exceptional for $A$. Hence, in case $\bullet A$, MP should not be applied to conditionals with antecedent $A$. The following restricted MP realizes this idea.

$$
\vdash((A \rightsquigarrow B) \wedge A \wedge \neg \bullet A) \supset B
$$

Due to the restriction, MP is only applied in case we are able to derive that the factual information is not exceptional with respect to $A$, i.e., $\neg \bullet A$. The following is an immediate consequence of [rMP] and the core properties:

$$
\vdash(A \wedge B \wedge(B \rightsquigarrow \neg A)) \supset \bullet B
$$


The antecedent of [Spe1] expresses that the default $B \rightsquigarrow \neg A$ is factually overridden since $A$ is the case. If the factual information describes atypical circumstances for $A$ and $A \rightsquigarrow B$, then we also have atypical circumstances for $B$, since after all $A$ is at least as specific as $B$. This motivates the following axiom: ${ }^{8}$

$$
\vdash(\bullet A \wedge(A \rightsquigarrow B)) \supset \bullet B
$$

Fact 1. [rMP], [Inh] and the core properties entail

$$
\vdash(A \wedge(A \rightsquigarrow B \rightsquigarrow C) \wedge(A \rightsquigarrow \neg C)) \supset \bullet B
$$

The antecedent of [Spe2] describes a case of specificity: the default $B \rightsquigarrow C$ is overridden by the more specific default $A \rightsquigarrow \neg C$ and the fact $A$. Let us take a look at a proof fragment for our example:

$\begin{array}{lll}1 & p \rightsquigarrow b & \text { PREM } \\ 2 & b \rightsquigarrow f & \text { PREM } \\ 3 & p \rightsquigarrow \neg f & \text { PREM } \\ 4 & p & \text { PREM } \\ 5 & b & \text { PREM } \\ 6 & \bullet b & 1,2,3,4 \text {; Spe2 }\end{array}$

Due to the fact that $\bullet b$ is derived at line 6 , our restriction prevents MP of being applicable to $b$ and $b \rightsquigarrow f$ in order to derive $f$. Indeed, due to $p$ we are in atypical circumstances with respect to $b$. This is for instance witnessed by the fact that by the core properties $b \rightsquigarrow \neg p$ is derivable from our premise set, and $p$ is a premise.

Note that something is still missing in order to model default inferencing properly. Due to the restricted MP we are able to block MP from being applied to excepted antecedents. However, we lack the ability to apply MP to $p \rightsquigarrow \neg f$ and $p$ since we miss $\neg \bullet p$. This can be tackled by applying MP conditionally. More specifically, MP is applied to $A \rightsquigarrow B$ and $A$ on the condition that the antecedent $A$ can be assumed to be not excepted, i.e., on the condition that $\bullet A$ can be assumed not to be the case. This is technically realized by means of adaptive logics.

I will introduce adaptive logics formally in Section 4 but let me sketch the main idea already now. In order to rewrite the proof above in the style of adaptive logics, we need to add a fourth column containing sets of so-called abnormalities. In our case abnormalities are of the form $\bullet A$.

8. The name $[\mathrm{Inh}]$ indicates that the property of being exceptional is inherited along $\rightsquigarrow-$ paths. 


$\begin{array}{rlll}1 & b \rightsquigarrow f & \text { PREM } & \emptyset \\ 2 & p \rightsquigarrow b & \text { PREM } & \emptyset \\ 3 & p \rightsquigarrow \neg f & \text { PREM } & \emptyset \\ 4 & b & \text { PREM } & \emptyset \\ 5 & p & \text { PREM } & \emptyset \\ 76 & f & 1,4 \text { RC } & \{\bullet b\} \\ 7 & \bullet b & 1,2,3,5 ; \text { Spe2 } & \emptyset \\ 8 & \neg f & 3,5 ; \text { RC } & \{\bullet p\}\end{array}$

At lines 6 and $8 \mathrm{MP}$ is applied conditionally (indicated by RC for "rule conditional"). For instance at line 8 the condition is $\{\bullet p\}$. In other words, MP is applied to $p$ and $p \rightsquigarrow \neg f$ on the condition that $p$ can be assumed to be not excepted. Note that if $\neg \bullet p$ would be derivable, we would be able to apply [rMP] to $p, p \rightsquigarrow \neg f$ and $\neg \bullet p$ in order to detach $\neg f$. However, $\neg \bullet p$ is not derivable. Nevertheless, adaptive logics offer the option to apply MP conditionally. Similarly, at line 6 MP is applied to $b \rightsquigarrow f$ and $b$ on the condition that $b$ is not excepted. However, at line $7, \bullet b$ is derived. Note that at this point line 6 is marked by 7 . The idea is that lines with "unsafe" conditions are marked and the formulas in the second column of marked lines are not considered as being derived. Of course, since $f$ is derived on the condition that $b$ is not excepted, this very condition cannot be considered safe anymore as soon as we derive that $b$ is excepted at line 7 . There are two adaptive strategies that specify what it exactly means that a condition of a line is "unsafe". For instance in case of the so-called reliability strategy a line is marked at a given stage of the proof in case a member of its condition has been derived as part of a minimal disjunction of abnormalities (in our case a disjunction of formulas preceded by a •) on the condition $\emptyset .{ }^{9}$ Minimality means that no sub-formula of the disjunction has been derived. Since $\bullet p$ is not derivable as part of a disjunction of abnormalities, line 8 is not going to be marked. There is obviously no reason to treat its condition as unsafe.

In the following sections I will realize the idea that was informally presented in this section. First, in Section 4, I introduce adaptive logics. Then, in Section 5, the adaptive logics for conditionally applying MP will be defined.

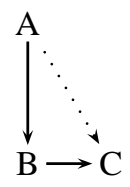

(a)

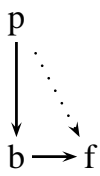

(b)

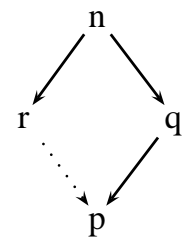

(c)

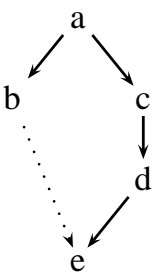

(d)

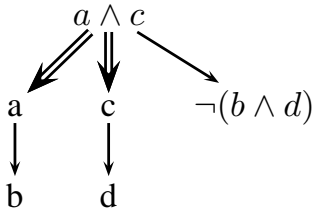

(e)

Figure 1: Illustrations and Examples

9. A more precise notion of what it means that a condition is "unsafe" will be given in the next section by means of a marking definition. 


\section{Adaptive logics}

An adaptive logic AL in standard format is a triple consisting of (i) a lower limit logic (henceforth LLL), which is a reflexive, transitive, monotonic, and compact logic that has a characteristic semantics and contains CL (classical logic), (ii) a set of abnormalities $\Omega$, characterized by a (possibly restricted) logical form, and (iii) an adaptive strategy. Formulating an adaptive logic in the standard format provides the logic with all of the important meta-theoretic features, such as soundness and completeness (as is shown in (Batens, 2007)).

In the following we use $\varphi$ and $\psi$ as meta-variables for well-formed formulas of a given language. The proof dynamics is governed by a marking definition for proof lines. The fact that the proofs are of a dynamic nature makes adaptive logics very useful for the modeling of defeasible reasoning, since a formula derivable at one stage of the proof may turn out not to be derivable at a later stage. A line of a proof consists of a line number, a formula, a justification, and a condition. Conditions are sets of abnormalities. We abbreviate $\bigvee_{\varphi \in \Delta} \varphi$ by $\operatorname{Dab}(\Delta)$ for some finite set $\Delta$ of abnormalities signifying the "disjunction of abnormalities in $\Delta$ ". Adaptive proofs are characterized by the following generic rules:

PREM If $\varphi \in \Gamma:$

$\mathrm{RU} \quad$ If $\varphi_{1}, \ldots, \varphi_{n} \vdash_{\text {LLL }} \psi:$

If $\varphi_{1}, \ldots, \varphi_{n} \vdash$ LLL $\psi$

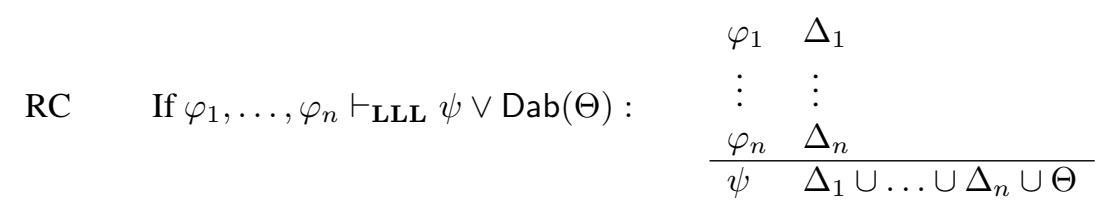

$\varphi_{1} \Delta_{1}$

$\vdots \quad \vdots$

\begin{tabular}{ll}
$\varphi_{n}$ & $\Delta_{n}$ \\
\hline$\psi$ & $\Delta_{1} \cup \ldots \cup \Delta_{n}$
\end{tabular}

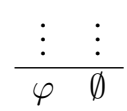

RU says that if a formula $\psi$ is derived on a line $l$ by means of the LLL from $\varphi_{1}, \ldots, \varphi_{n}$ that are derived on conditions $\Delta_{1}, \ldots, \Delta_{n}$, then these conditions are carried forward to line $l$. Note, that by RU all LLL-rules are valid in AL and thus all LLL-consequences are AL-consequences.

The essential strength of adaptive logics comes with the rule RC. It enables us to derive formulas conditionally. Also for applications of RC conditions are carried forward, as it was the case for RU. RC is used to derive $\psi$ on the condition $\Theta$ if, by $\mathbf{L L L}, \psi \vee \operatorname{Dab}(\Theta)$ is derivable. The idea is to assume that the abnormal members of $\Theta$ are false, in which case $\psi$ has to be true. Of course, there are circumstances in which this assumption cannot be maintained. In such cases lines with "unsafe" conditions 
are marked. Before I come to the marking definition, some more notions have to be introduced.

Stages of proofs are lists of lines obtained by applications of the generic rules above (with the usual understanding that the justification of a line should only refer to lines preceding it in the list). The empty list will be considered as stage 0 of every proof. Where $s$ is a stage, $s^{\prime}$ is an extension of $s$ iff all lines that occur in $s$ occur in the same order in $s^{\prime}$. A (dynamic) proof is a sequence of stages.

Given a premise set $\Gamma, \operatorname{Dab}(\Delta)$ is a minimal Dab-formula at stage $s$ of the proof from $\Gamma$, iff it is the formula of a line with condition $\emptyset$ and no $\operatorname{Dab}\left(\Delta^{\prime}\right)$, where $\Delta^{\prime} \subset$ $\Delta$, is the formula of a line with condition $\emptyset$. Where $\operatorname{Dab}\left(\Delta_{1}\right), \operatorname{Dab}\left(\Delta_{2}\right), \ldots$ are the minimal Dab-formulas at stage $s$, we define the set of unreliable formulas at stage $s$, $U_{s}(\Gamma)=\Delta_{1} \cup \Delta_{2} \cup \ldots$ We call the minimal Dab-formulas derivable with LLL, the minimal Dab-consequences. Where $\operatorname{Dab}\left(\Delta_{1}\right), \operatorname{Dab}\left(\Delta_{2}\right), \ldots$ are the minimal Dabconsequences, we define the set of unreliable formulas, $U(\Gamma)=\Delta_{1} \cup \Delta_{2} \ldots$.

It is the job of the marking definition to determine if lines are "in" or "out" of the proof at a certain stage, i.e., to govern the dynamics of the proof procedure. For the reliability strategy lines are marked which have unreliable formulas in their condition.

Definition 1 (Marking for Reliability). Line $i$ is marked at stage $s$ iff, where $\Delta$ is its condition, $\Delta \cap U_{s}(\Gamma) \neq \emptyset$.

For the minimal abnormality strategy a few more notions need to be introduced. A choice set of $\Sigma=\left\{\Delta_{1}, \Delta_{2}, \ldots\right\}$ is a set that contains an element out of each member of $\Sigma$. A minimal choice set of $\Sigma$ is a choice set of $\Sigma$ of which no proper subset is a choice set of $\Sigma$. Where, for a premise set $\Gamma, \operatorname{Dab}\left(\Delta_{1}\right), \operatorname{Dab}\left(\Delta_{2}\right), \ldots$ are the minimal Dab-formulas at stage $s, \Phi_{s}(\Gamma)$ is the set of the minimal choice sets of $\left\{\Delta_{1}, \Delta_{2}, \ldots\right\}$.

Definition 2 (Marking for minimal abnormality). Line $i$ is marked at stage $s$ iff, where $\varphi$ is derived on the condition $\Delta$ at line $i$,

(i) there is no $\Delta^{\prime} \in \Phi_{s}(\Gamma)$ such that $\Delta^{\prime} \cap \Delta=\emptyset$, or

(ii) for some $\Delta^{\prime} \in \Phi_{s}(\Gamma)$, there is no line at which $\varphi$ is derived on a condition $\Theta$ for which $\Delta^{\prime} \cap \Theta=\emptyset$.

Note that a line may be marked at a stage $s$ of the proof, but be unmarked at a later stage $s^{\prime}$. Indeed, even if $\operatorname{Dab}(\Delta)$ is a minimal Dab-formula at stage $s$, we may be able to derive $\operatorname{Dab}\left(\Delta^{\prime}\right)$ where $\Delta^{\prime} \subset \Delta$ at a later stage $s^{\prime}$. This may lead to an alteration of the unreliable formulas or/and the minimal choice sets and thus to changes in the marking of lines. In order to define the consequence set of an adaptive logic, a stable criterion for derivability is offered by the following definition.

Definition 3. $\varphi$ is finally derived from $\Gamma$ on line $i$ of a proof at a finite stage $s$ iff (i) $\varphi$ is the second element of line $i$, (ii) line $i$ is not marked at stage $s$ and (iii) every extension of the proof in which line $i$ is marked may be further extended in such a way that line $i$ is unmarked. 
$\Gamma \vdash_{\mathbf{A L}} \varphi(\varphi$ is finally $\mathbf{A L}$-derivable from $\Gamma)$ iff $\varphi$ is finally derived on a line of a proof from $\Gamma$.

Let us have a look at the semantics. The idea behind the minimal abnormality strategy is that only the models (of a given premise set) which validate a minimal set of abnormalities are taken into account. For the reliability strategy only models are considered whose abnormal part is a subset of the set of unreliable formulas.

Definition 4. An LLL-model $M$ is reliable iff $\operatorname{Ab}(M) \subseteq U(\Gamma)$, where $\operatorname{Ab}(M)=_{\mathrm{df}}$ $\{\varphi \mid M \models \varphi\} \cap \Omega$. An LLL-model $M$ of $\Gamma$ is minimal abnormal iff there is no LLL-model $M^{\prime}$ of $\Gamma$ such that $\mathrm{Ab}\left(M^{\prime}\right) \subset \mathrm{Ab}(M)$.

$\Gamma \Vdash_{\text {AL }} \varphi$ ( $\varphi$ is an AL-semantic consequence of $\Gamma$ ) iff $\varphi$ is verified by, depending on the strategy, all reliable models resp. all minimal abnormal LLL-models of $\Gamma$.

The following completeness and soundness result is valid for all adaptive logics AL in standard format (as shown in (Batens, 2007)): $\Gamma \vdash_{\text {AL }} \varphi$ iff $\Gamma \vdash_{\text {AL }} \varphi$.

\section{Applying Modus Ponens Conditionally}

As discussed in Section 3, we use a unary operator ' $\bullet$ ' in order to label propositional formulas for which MP should be blocked. These are propositions that are excepted by the information given in the premises. That is to say, the factual information at hand describes unusual circumstances concerning them.

We have seen that $b$, "Tweety is a bird.", is excepted if also $p$, "Tweety is a penguin.", is given. The second proposition describes an exceptional context for the first one due to the conditionals $b \rightsquigarrow f, p \rightsquigarrow b$ and $p \rightsquigarrow \neg f$ where $f=$ "Tweety flies". Thus, $f$ should not be detached from $b \rightsquigarrow f$ and $b$ if $p$ is the case: $b \rightsquigarrow f$ is overridden by the more specific $p \rightsquigarrow \neg f$.

The following fact shows that in various cases of specificity the least specific arguments are excepted.

Fact 2. The core properties, [rMP] and [Inh] imply [Spe1], [Spe2] and the following:

$$
\begin{array}{rr}
\text { If } \vdash A \supset B, \text { then } \vdash(A \wedge(B \rightsquigarrow C) \wedge(A \rightsquigarrow \neg C)) \supset \bullet B & \text { [sSpe] } \\
\vdash\left(A \wedge\left(A \rightsquigarrow B_{1} \rightsquigarrow \ldots \rightsquigarrow B_{n} \rightsquigarrow C\right) \wedge(A \rightsquigarrow \neg C)\right) \supset \bullet B_{n} & \text { [SpeG] } \\
\vdash\left(A \wedge\left(A \rightsquigarrow B_{1} \rightsquigarrow \ldots \rightsquigarrow B_{n} \rightsquigarrow D\right) \wedge\right. & \\
\left.\left(A \rightsquigarrow C_{1} \rightsquigarrow \ldots \rightsquigarrow C_{m} \rightsquigarrow \neg D\right) \wedge\left(B_{n} \rightsquigarrow \ldots \rightsquigarrow C_{m}\right)\right) \supset \bullet C_{m} & \text { [PreE] } \\
\text { If } \vdash \neg \bigwedge_{I} D_{i}, \text { then } \vdash\left(A \wedge \bigwedge_{I}\left(A \rightsquigarrow \ldots \rightsquigarrow B_{i} \rightsquigarrow D_{i}\right)\right) \supset \bigvee_{I} \bullet B_{i} & {[\text { Conf] }}
\end{array}
$$


[SpeG] is a generalization of [Spe2] (see Figure 2b). The preemption rule [PreE] is a further generalization (see Figure 2c). ${ }^{10}$ [Conf] shows that if there are multiple conflicting arguments $A \rightsquigarrow \ldots \rightsquigarrow B_{i} \rightsquigarrow D_{i}$ then at least one of the $B_{i}$ 's is excepted.

Let in the remainder $\mathbf{L p}$ be the base logic $\mathbf{L}$ enriched by [rMP] and [Inh]. In this paper we will focus on base logics $\mathbf{L} \in\{\mathbf{P}, \mathbf{R}\}$ (see Section 2).

Definition 5. We define $\mathbf{D L} \mathbf{p}^{\mathbf{x}}$ where $\mathbf{x} \in\{\mathbf{r}, \mathbf{m}\}$ as an adaptive logic in standard format by the following triple:

- the lower limit logic is $\mathbf{L p}$,

- the set of abnormalities is $\Omega=\{\bullet A \mid A \in \mathcal{W}\}$,

- the strategy is either reliability (for $\mathbf{D L} \mathbf{p}^{\mathbf{r}}$ ) or minimal abnormality (for DLp $\left.{ }^{\mathbf{m}}\right)$.

To adaptively interpret premise sets "as normally as possible" means in our case to interpret the propositional formulas as not being excepted whenever possible, i.e., whenever this is consistent with the given premises. In turn, this allows us to apply MP as much as possible since the additional antecedents of [rMP], $\neg \bullet A$, are validated as much as possible. Note that due to [rMP] we have

$$
\vdash_{\mathbf{L} \mathbf{p}}(A \wedge(A \rightsquigarrow B)) \supset(B \vee \bullet A)
$$

Hence, by RC, $B$ is derivable from $A$ and $A \rightsquigarrow B$ on the condition $\{\bullet A\}$.

The (object-level) proofs presented in the following examples are for both adaptive logics, $\mathbf{D} \mathbf{L} \mathbf{p}^{\mathbf{r}}$, and $\mathbf{D} \mathbf{L} \mathbf{p}^{\mathbf{m}}$, if not specified differently. I presume that $\mathbf{L} \in\{\mathbf{P}, \mathbf{R}\}$. Let us take a look at a simple case of specificity.

Example 2. We equip the conditional knowledge base in Example 1 (see Figure 1b) with the factual knowledge $\{p\}$.

$\begin{array}{llllrlll}1 & p \rightsquigarrow b & \text { PREM } & \emptyset & 5 & b & 1,4 ; \mathrm{RC} & \\ 2 & b \rightsquigarrow f & \text { PREM } & \emptyset & 86 & f & 2,5 ; \mathrm{RC} & \\ 3 & p \rightsquigarrow \neg f & \text { PREM } & \emptyset & 7 & \neg f & 3,4 ; \mathrm{RC} & \{\bullet p\} \\ 4 & p & \text { PREM } & \emptyset & 8 & \bullet b & 1,2,3,4 ; \mathrm{RU} & \emptyset\end{array}$

At line 5, MP is applied to $p \rightsquigarrow b$ and $p$ on the condition $\{\bullet p\} .{ }^{11}$ Similar conditional applications take place at lines 6 and 7. The desired $\neg f$ and $b$ are (finally) derivable since the condition, $\bullet p$, is not part of any minimal Dab-consequence. Moreover, MP is blocked from $b \rightsquigarrow f$ and $b$ since at line $8, \bullet b$ is derived and hence line 6 is marked.

Example 3. Let us have a look at conflicting conditionals by means of the Nixon Diamond (see Figure 1c) with the factual knowledge $\{n\}$ and the usual reading of $q$ as 'being a Quaker', $r$ 'being a Republican' and $p$ as 'being a pacifist'.

10. Preemption plays an important role in the research on inheritance networks (see (Horty, 1994)).

11. This is accomplished by means of the generic rule RC as defined on page 11 . 


$\begin{array}{lllrrlll}1 & n \rightsquigarrow q & \text { PREM } & \emptyset & 6 & q & 1,5 ; \mathrm{RC} & \{\bullet n\} \\ 2 & n \rightsquigarrow r & \text { PREM } & \emptyset & 7 & r & 2,5 ; \mathrm{RC} & \{\bullet n\} \\ 3 & q \rightsquigarrow p & \text { PREM } & \emptyset & { }^{10} 8 & p & 3,6 \text {; RC } & \{\bullet n, \bullet q\} \\ 4 & r \rightsquigarrow \neg p & \text { PREM } & \emptyset & 109 & \neg p & 4,7 ; \mathrm{RC} & \{\bullet n, \bullet r\} \\ 5 & n & \text { PREM } & \emptyset & 10 & \bullet q \vee \bullet r & 1,2,3,4,5 ; \mathrm{RU} & \emptyset\end{array}$

The logic proceeds as expected: $r$ and $q$ are derivable while the derivations of $p$ and $\neg p$ get marked for both strategies. Note that the condition of line 6 and 7 , namely $\{\bullet n\}$, is not part of any minimal Dab-consequence. In order to make the example more interesting let us introduce two more conditionals: $q \rightsquigarrow e$ and $r \rightsquigarrow e$ where $e$ represents for instance 'being politically motivated'.

$\begin{array}{llll}11 & q \rightsquigarrow e & \text { PREM } & \emptyset \\ 12 & r \rightsquigarrow e & \text { PREM } & \emptyset \\ 13 & e & 6,11 ; \mathrm{RC} & \{\bullet n, \bullet q\} \\ 14 & e & 7,12 ; \mathrm{RC} & \{\bullet n, \bullet r\}\end{array}$

By the reliability strategy lines 13 and 14 are marked (due to the fact that $\bullet q \vee$ - $r$ at line 10 is a minimal Dab-consequence). They are not marked by the minimal abnormality strategy, since the minimal choice sets at line 14 are $\{\bullet q\}$ and $\{\bullet r\}$. It is easy to see that there is no way to extend the proof in a way such that lines 13 and 14 are marked according to the minimal abnormality strategy. This shows that the reliability strategy models a more skeptical reasoning in comparison to the bolder reasoning type modeled by the minimal abnormality strategy.

We have a similar scenario for the example depicted in Figure 2e. By the minimal abnormality strategy $p$ is derivable given the factual knowledge $x$. It is not derivable by the reliability strategy.

Propositions such as $p$ in Figure 2e are commonly dubbed "floating conclusions". There is a vivid debate about whether such propositions should be accepted. ${ }^{12}$ Instead of trying to have the final word on the discussion I want to point out that, as the example shows, the minimal abnormality strategy detaches floating conclusions, while the more skeptical reliability strategy rejects them. Different applications may ask for different strategies. The credulous character of the minimal abnormality strategy makes it interesting for applications in which "the value of drawing conclusions is high relative to the costs involved if some of those conclusions turn out not to be correct." ((Horty, 1994), p. 14). The reliability strategy on the other hand is, due to its more skeptical character, better "when the cost of error rises" (ibid.).

Example 4. Let our knowledge base be $\Gamma_{4}=\left\{a_{i} \rightsquigarrow a_{i+1} \mid 1 \leq i<n\right\}$ (see Figure 2a) with factual knowledge $\left\{a_{1}\right\}$. Note that $\Gamma_{4} \nvdash_{\mathbf{P}} a_{1} \rightsquigarrow a_{j}$ and $\Gamma_{4} \nvdash_{\mathbf{R}} a_{1} \rightsquigarrow a_{j}$ where $2<j \leq n$. However, our adaptive logics are able to detach all the $a_{i}$ 's:

12. While Ginsberg (Ginsberg, 1994), and Makinson and Schlechta (Makinson et al., 1991) argue for the acceptance, Horty (Horty, 2002) argues against it. 


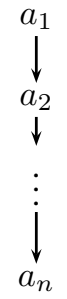

(a)

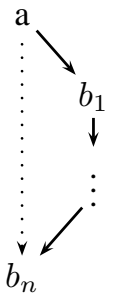

(b)

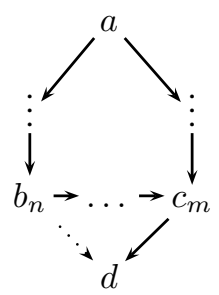

(c)

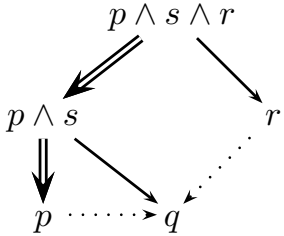

(d)

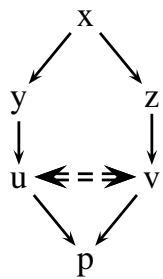

(e)

Figure 2: Illustrations and Examples

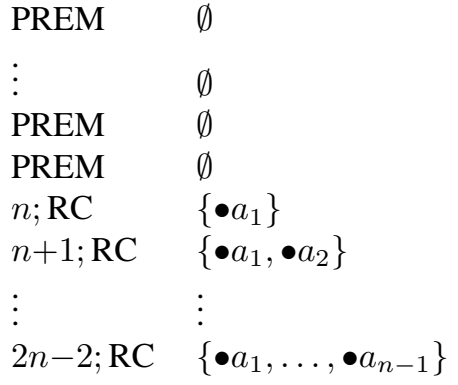

Obviously none of the lines $n+1, \ldots, 2 n-1$ can be marked by extending the proof. The fact that $\Gamma_{4} \cup\left\{a_{1}\right\} \vdash_{\mathbf{D L}_{\mathbf{p}}} a_{i}$, where $i \leq n, \mathbf{x} \in\{\mathbf{r}, \mathbf{m}\}$ and $\mathbf{L} \in\{\mathbf{P}, \mathbf{R}\}$, while $\Gamma_{4} \nvdash_{\mathbf{L}} a_{1} \rightsquigarrow a_{i}$ demonstrates that our handling of MP overcomes certain weaknesses of the core logic in terms of the handling of transitive relations among conditionals.

Example 5. Let our factual knowledge be $a . b_{1}, \ldots, b_{n-1}$ and $\neg b_{n}$ are derivable from the knowledge base depicted in Figure $2 b$ by means of $\mathbf{D P} \mathbf{p}^{\mathbf{x}}$ (where $\mathbf{x} \in\{\mathbf{r}, \mathbf{m}\}$ ). We obtain e.g. the Dab-formula $\bullet b_{n-1}$ (by [SpeG]) and $\bullet b_{n}$ (by means of the former and [Inh]). Note that no $\bullet b_{i}$ where $i<n-1$ is derivable as a part of a minimal Dab-consequence. Hence we can iteratively apply Modus Ponens conditionally to $a \rightsquigarrow b_{1}$ and $b_{i} \rightsquigarrow b_{i+1}$ where $i<n-1$ in such a way that the corresponding lines are unmarked. Note that $a \rightsquigarrow b_{n-1}$ is neither a $\mathbf{P}$-consequence nor a $\mathbf{R}$-consequence of the given premises, nor is it derivable by means of Rational Closure. However, it is entailed by $\mathbf{P}_{\min } .{ }^{13}$

The situation is slightly different in $\mathbf{D R} \mathbf{p}^{\mathbf{x}}$ : besides $\bullet b_{n-1}$ and $\bullet b_{n}$ also $\bullet b_{n-2} \vee$ $\bullet \neg b_{n-2}$ is $\mathbf{R p}$-derivable from the premises. ${ }^{14}$ It is easy to see that due to this $b_{n-1}$ is not $\mathbf{D R} \mathbf{p}^{\mathbf{x}}$-derivable since the only means of deriving $b_{n-1}$ from the given premises

13. Note that in case we do not add $a \not \leftrightarrow \perp$ to our premises, $\mathbf{P}_{\min }$ is rather rigorous and also entails $a \rightsquigarrow \perp$.

14. The reason is as follows. Suppose first that $b_{n-1} \not \Varangle \neg b_{n-2}$. In this case by means of [RM] and since $b_{n-1} \rightsquigarrow b_{n}$ also $\left(b_{n-1} \wedge b_{n-2}\right) \rightsquigarrow b_{n}$. By [RT] and since $b_{n-2} \rightsquigarrow b_{n-1}$, $b_{n-2} \rightsquigarrow b_{n}$. But then since $a, a \rightsquigarrow \neg b_{n}$ and $a \rightsquigarrow \ldots \rightsquigarrow b_{n-2} \rightsquigarrow b_{n}$, by [SpeG], $\bullet b_{n-2}$. Now suppose $b_{n-1} \rightsquigarrow \neg b_{n-2}$. Since $\bullet b_{n-1}$ we get $\bullet \neg b_{n-2}$ by [Inh]. Altogether, $\bullet b_{n-2} \vee \bullet \neg b_{n-2}$. Note that this argument does not hold in $\mathbf{P p}$ since it makes essentially use of [RM]. 
is by detaching it from the conditional $b_{n-2} \rightsquigarrow b_{n-1}$ on the condition $\left\{\bullet b_{n-2}\right\}$. Yet, due to the minimal Dab-consequence $\bullet b_{n-2} \vee \bullet \neg b_{n-2}$ any such attempt gets marked in the proof. However, if we add the premise $b_{n-2} \not b_{n}$ we get the consequences $b_{i}$ for all $i<n$ and $\neg b_{n}$ just as for $\mathbf{D P} \mathbf{p}^{\mathbf{x}}$. It is easy to see that in this case $\bullet b_{n-2}$ is not anymore part of any minimal Dab-consequence.

Similarly, $b_{1}, \ldots, b_{n}, c_{1}, \ldots, c_{m}$ and $\neg d$ are $\mathbf{D P} \mathbf{p}^{\mathbf{x}}$-derivable from the knowledge base depicted in Figure 2c. Analogous to the previous paragraph we need to add another premise, e.g. $c_{m-1} \not \ngtr a$, in order to get the same consequences for $\mathbf{D R} \mathbf{p}^{\mathbf{x}}$. The proofs are simple and left to the reader.

Example 6. Let us take a look at a variant of the Nixon Diamond (Figure 1d) by means of the logic $\mathbf{D P} \mathbf{p}^{\mathbf{x}}$ (where $\mathbf{x} \in\{\mathbf{r}, \mathbf{m}\}$ ):

$\begin{array}{lllrrlll}1 & a \rightsquigarrow b & \text { PREM } & \emptyset & 7 & b & 1,6 ; \mathrm{RC} & \{\bullet a\} \\ 2 & a \rightsquigarrow c & \text { PREM } & \emptyset & 128 & \neg e & 3,7 ; \mathrm{RC} & \{\bullet a, \bullet b\} \\ 3 & b \rightsquigarrow \neg e & \text { PREM } & \emptyset & 9 & c & 2,6 ; \mathrm{RC} & \{\bullet a\} \\ 4 & c \rightsquigarrow d & \text { PREM } & \emptyset & 10 & d & 4,9 ; \mathrm{RC} & \{\bullet a, \bullet c\} \\ 5 & d \rightsquigarrow e & \text { PREM } & \emptyset & 1211 & e & 5,10 ; \mathrm{RC} & \{\bullet a, \bullet c, \bullet d\} \\ 6 & a & \text { PREM } & \emptyset & 12 & \bullet b \vee \bullet d & 1-6 ; \mathrm{RU} & \emptyset\end{array}$

Note that neither is $a \rightsquigarrow d$ derivable by the core properties nor is it in the Rational Closure, nor is it entailed by Conditional Entailment. ${ }^{15}$ Thus, in the given example our logic handles the transitive relations between defaults better than these systems, since (with both strategies) $d$ is derivable following argument $a \rightsquigarrow c \rightsquigarrow d$. Furthermore, as desired, neither $e$ nor $\neg e$ is derivable since there are conflicting arguments concerning $e$ and $\neg e$.

The situation is different in $\mathbf{D} \mathbf{R} \mathbf{p}^{\mathbf{x}}$ since by means of $\mathbf{R} \mathbf{p}$ also the minimal Dabconsequence $\bullet c \vee \bullet \neg c$ is derivable. ${ }^{16}$ Hence, there $d$ at line 10 is not finally derivable. However, by adding $c \not \neg \neg a$ to the premises also $d$ is a $\mathbf{D R} \mathbf{p}^{\mathbf{x}}$-consequence of this premise set.

Example 7. We take a look at Figure 1e with factual knowledge $\{a \wedge c\}$. This example illustrates a more complex case of specificity.

15. Note that $\mathbf{P}_{\text {min }}$ entails $a \rightsquigarrow d$ and moreover $a \rightsquigarrow \perp$ (in case we do not manually add $a \not \leftrightarrow \perp$ to the premises, see also footnote 13).

16. The reason is as follows. Suppose $\neg \bullet c$. Suppose (i) $b \rightsquigarrow \neg a$. Since also $a, a \rightsquigarrow b$ and $a \rightsquigarrow a$ (by [ID]) we get $\bullet b$ by [Spe2]. Assume $b \not \hookrightarrow \neg c$. By [RM], $(b \wedge c) \rightsquigarrow \neg a$. Since also $a, a \rightsquigarrow a$ and $a \rightsquigarrow(b \wedge c)$ (by [CC], $a \rightsquigarrow b$ and $a \rightsquigarrow c$ ) we have $\bullet(b \wedge c)$ by [Spe2]. By [Inh] and [CI] also $\bullet c,-$ a contradiction. Hence, $b \rightsquigarrow \neg c$. By [Inh] $\bullet \neg c$. Now suppose (ii) $b \ngtr \neg \neg a$. Since $b \rightsquigarrow \neg e$ by [RM] $(a \wedge b) \rightsquigarrow \neg e$. Since $a \rightsquigarrow b$ by [RT] $a \rightsquigarrow \neg e$. By the latter, $a, \neg \bullet c$ and $a \rightsquigarrow c$ we have $c \not \rightsquigarrow e$ due to [Spe2]. By $a, a \rightsquigarrow \neg e$ and $a \rightsquigarrow c \rightsquigarrow d \rightsquigarrow e$ we have $\bullet d$ due to [SpeG]. Assume $d \not \neg \neg c$. Then by [RM] $(c \wedge d) \rightsquigarrow e$ and by [RT], $c \rightsquigarrow e,-$ a contradiction. Hence $d \rightsquigarrow \neg c$ and by [Inh], $\bullet \neg c$. Altogether we get $\bullet c \vee \bullet \neg c$. 


$\begin{array}{lll}a \wedge c \rightsquigarrow \neg(b \wedge d) & \text { PREM } & \emptyset \\ a \rightsquigarrow b & \text { PREM } & \emptyset \\ c \rightsquigarrow d & \text { PREM } & \emptyset \\ a \wedge c & \text { PREM } & \emptyset \\ \neg(b \wedge d) & 1,4 ; \mathrm{RC} & \{\bullet(a \wedge c)\} \\ b & 2,4 ; \mathrm{RC} & \{\bullet a\} \\ d & 3,4 ; \mathrm{RC} & \{\bullet c\} \\ \bullet a \vee \bullet c \vee \bullet(a \wedge c) & 1,2,3,4 ; \mathrm{RU} & \emptyset \\ \bullet a \vee \bullet c & 8 ; \mathrm{RU} & \emptyset \\ b \vee d & 6 ; \mathrm{RU} & \{\bullet a\} \\ b \vee d & 7 ; \mathrm{RU} & \{\bullet c\}\end{array}$

Line 9 follows from line 8 in view of [Inh] and [CI]. By the reliability strategy lines 10 and 11 are marked since both, $\bullet a$ and $\bullet c$, are unreliable formulas. Not so by the minimal abnormality strategy, since $b \vee d$ is derivable on both conditions, $\{\bullet a\}$ and $\{\bullet c\}$ (see Definition 1).

This example is interesting also in another respect. It features a more complex type of specificity. While none of the arguments $\mathrm{A}_{1}=(a \wedge c) \rightsquigarrow a \rightsquigarrow b$ and $\mathrm{A}_{2}=$ $(a \wedge c) \rightsquigarrow c \rightsquigarrow d$ suffices in its own respect to cause a case of specificity with $(a \wedge c) \rightsquigarrow \neg(b \wedge d)$, both taken together do. Indeed, if we follow both lines of argument, $\mathrm{A}_{1}$ and $\mathrm{A}_{2}$, we arrive at $b$ and $d$. However, the conjunction $b \wedge d$ contradicts $\neg(b \wedge d)$. Thus, $a \wedge c \rightsquigarrow \neg(b \wedge d)$ overrides the joint application of arguments $\mathrm{A}_{1}$ and $\mathrm{A}_{2}$ (see also the illustration in Figure 1e).

Both, minimal abnormality and reliability strategy, validate $\neg(b \wedge d)$. Again, if we apply reliability we take a more skeptical route concerning $A_{1}$ and $A_{2}$, since both arguments are considered as being unreliable and thus neither argument is validated: we neither derive $b$, nor $d$, nor $b \vee d$. Minimal abnormality however validates one of the two arguments. Indeed, taken isolated from each other, neither $A_{1}$ nor $A_{2}$ is overridden by $a \wedge c \rightsquigarrow \neg(b \wedge d)$. Thus, the credulous reasoning provided by the minimal abnormality strategy validates $b \vee d$ and $\neg(b \wedge d)$.

Example 8. Given the factual knowledge $p \wedge s \wedge r$ and the defaults depicted in Figure $2 \mathrm{~d}$ we have the minimal Dab-consequence $\bullet(p \wedge s) \vee \bullet r$. That shows that neither $q$ nor $\neg q$ is derivable. This is intuitive as pointed out by Geffner and Pearl in (Geffner $e t$ al., 1992) since there are no reasons to prefer argument $(p \wedge s) \rightsquigarrow q$ over $r \rightsquigarrow \neg q$ or vice versa. Note however that the counter-intuitive $(p \wedge s \wedge r) \rightsquigarrow q$ is in the Rational and Lexicographic Closure, and it is entailed by the maximum entropy approach. ${ }^{17}$

\section{Discussion}

In this discussion section I will point out some advantages of the presented logics, also in comparison with other systems from the literature. Moreover I will comment on some other related and interesting points which were not mentioned so far.

17. It is not entailed by $\mathbf{P}_{\text {min }}$ in case we add $(p \wedge s \wedge r) \not \leftrightarrow \rightarrow$. 


\subsection{Some advantages of the adaptive approach}

Adaptive logics offer a very generic framework enabling defeasible MP for conditional logics of normality since they can be applied to any conditional lower limit logic as long as it is reflexive, transitive, monotonic and compact. Depending on the application the reader is free to use any conditional logic of normality as LLL as long as it fulfills the mentioned requirements. Since adaptive logics have shown great unifying power in representing nonmonotonic, defeasible logics ${ }^{18}$, even conditional logics that do not fulfill the requirements may be represented by adaptive logics. ${ }^{19}$ By applying techniques of combining adaptive systems the framework developed in this paper may be applicable also in such cases. Furthermore, similar techniques as presented here for defeasible MP in the context of default reasoning can be applied to conditional deontic logics (see (Straßer, 2010)).

The meta-theory of adaptive logics in standard format is well-researched (see (Batens, 2007; Putte et al., 201X)). Many useful properties have been established generically. For instance, completeness and soundness of an adaptive logic are guaranteed by the completeness and soundness of its LLL, the consequence relation of an adaptive logic defines a fixed point and is cautious monotonic, etc.

Pollock distinguished in (Pollock, 1987) between two types of dynamics that characterize defeasible reasoning: one based on synchronic defeasibility and another one based on diachronic defeasibility. As I will discuss in the following, adaptive logics are able to model both of them.

The internal dynamics of defeasible reasoning is caused by diachronic defeasibility. Often achieving a better understanding of the information at hand forces us to withdraw certain inferences even in cases in which no new information is available. This is modeled by the dynamic proof theory of adaptive logics. For instance, if we (conditionally) apply MP to $b \rightsquigarrow f$ and $b$ but at a later moment also derive $p, p \rightsquigarrow \neg f$ and $p \rightsquigarrow b$ from the same premises, we revise the former derivation. In the adaptive proof the line at which MP has been applied to $b \rightsquigarrow f$ and $b$ is going to be marked and is hence considered not to be valid. Thus, while our insight in the given knowledge base - i.e., the premises - grows, we may consider revising some conclusions drawn before, especially if the knowledge base is of a complex nature. Hence, our treatment of common sense reasoning with factual information on the basis of conditional knowledge bases does not just reach intuitive results but the explication of the reasoning process itself is an integral part of the proof theory. This is an advantage compared to other systems which are able to model default inferencing such as Delgrande's (Delgrande, 1988), Lamarre's (Lamarre, 1993), or Geffner and Pearl's (Geffner et al., 1992).

Lamarre in (Lamarre, 1993) presents a powerful approach based on semantic selection procedures on the models of a given conditional base logic, where the facts valid

18. For the most recent survey see (Batens et al., 2009).

19. As has been shown, for instance, for Rational Closure in (Straßer, 2009). 
in all the selected models characterize the consequence set of his system. ${ }^{20}$ What is missing, however, is a syntactical approach corresponding to it that mirrors our common sense reasoning by its proof theory. Delgrande's system (Delgrande, 1988) is syntactical in nature. The idea here is to iteratively enrich the given factual knowledge by further contingent information in order to form so-called maximal contingent extensions. ${ }^{21}$ Special attention in building these extensions is given to cases of specificity: similar as in the presented approach, the world at hand is interpreted as non-exceptional as possible. Furthermore, in the construction of the extensions only relevant information is considered with respect to the knowledge base at hand. What is derivable by classical logic from these maximal contingent extensions corresponds to the factual consequences we draw via default reasoning. While Delgrande's assumptions concerning the normality of the actual world and his restriction to relevant information accord with a natural intuition concerning default reasoning, the way we arrive at the inferences by Delgrande's approach seems rather unnatural, i.e., the technical necessity to first built up all the maximal consistent factual extensions and then to infer from them by classical reasoning. This procedure does not model our actual default inferencing in an accurate way. Geffner and Pearl's Conditional Entailment has been already mentioned on Section 2. Although the authors provide a syntactic check-criterion for conditionally entailed propositions, they do not offer a derivational procedure that mirrors our actual reasoning processes such as the dynamic proofs of adaptive logics. ${ }^{22}$

As mentioned, another advantage of adaptive logics is their ability to deal with the synchronic defeasibility that causes the external dynamics of reasoning processes. Often with the introduction of new information we are forced to withdraw certain inferences. Again, the markings of the dynamic proofs are able to model cases of specificity and conflicting arguments which might be caused by new information. In contrast, in Lamarre's approach the arrival of new information forces us to re-initiate the semantic selection procedure, and, similarly, for Delgrande's account we have to re-construct the maximal contingent extensions. In the adaptive approach, despite the fact that new information might force us to withdraw certain conclusions, the proof dynamics model in an accurate way the fact that we continue reasoning facing new information instead of beginning the reasoning process again from scratch.

20. As discussed in Section 4, adaptive logics also employ semantic selections on the models of the LLL.

21. Delgrande introduces in fact two equivalent proposals in this paper. The other one, which I do not discuss above, is based on forming maximal consistent extensions of the conditional knowledge base at hand (in contrast to the maximal consistent extensions of the factual knowledge which I discuss here). Note, however, that a similar criticism applies to both approaches.

22. Computing Conditional Entailment is a pretty complex and challenging task. Hence, the authors only offer a computational approximation in terms of an assumption-based truth maintenance-like system (see (de Kleer, 1987)). 


\subsection{The Drowning Problem}

In Examples 4 and 6 it was demonstrated that the presented treatment of MP sometimes outgrows the abilities of the core system in terms of transitively closing $\rightsquigarrow$. However, there are limitations to it. To show this I extend Example 2 by a further conditional:

Example 9. We add to the conditionals of Example 2,b $\rightsquigarrow w$, where $w$ stands for "having wings". The proof of Example 2 is extended in the following way:

$$
\begin{array}{rlll}
9 & b \rightsquigarrow w & \text { PREM } & \emptyset \\
810 & w & 5,9 ; \operatorname{RC} & \{\bullet p, \bullet b\}
\end{array}
$$

Note that the conditional derivation of $w$ is not successful in the sense that it gets marked. This is due to the fact that $b$ is excepted since we have $p$ and $b \rightsquigarrow \neg p$. Indeed there is no way to derive $w$ from the given premises. This is also due to the fact that in $\mathbf{P}$ and $\mathbf{R}$ neither $p \rightsquigarrow w$ nor $(p \wedge b) \rightsquigarrow w$ is derivable (neither are they in the Rational Closure). Note that if one of the latter would be derivable, $w$ would be detachable from $p \rightsquigarrow w$ and $p$, or resp. $(p \wedge b) \rightsquigarrow w$ and $p \wedge b$. Thus, the limitation of the adaptive treatment of MP concerning excepted propositions mirrors a limitation of the base logic concerning conditional consequences.

This problem is commonly known as the Drowning Problem: suppose a default with antecedent $A$ is excepted, then all other defaults with antecedent $A$ are blocked from MP as well.

The first question to ask at this point is whether a "solution" to the drowning problem is at all desirable. Some scholars voice worries (see e.g. (Jeffry et al., 1994; Wobcke, 1995; Bonevac, 2003; Koons, 2009)). For instance, Koons asserts that there are good reasons why we should not apply MP to defaults with excepted antecedents. "Consider the following variant on the problem: birds fly, Tweety is a bird that doesn't fly, and birds have strong forelimb muscles. Here it seems we should refrain from concluding that Tweety has strong forelimb muscles, since there is reason to doubt that the strength of wing muscles is causally (and hence, probabilistically) independent of capacity for flight. Once we know that Tweety is an exceptional bird, we should refrain from applying other conditionals with Tweety is a bird as their antecedents, unless we know that these conditionals are independent of flight, that is, unless we know that the conditional with the stronger antecedent, Tweety is a nonflying bird, is also true." (see (Koons, 2009), Section 5.7)

Moreover, Lehmann in (Lehmann, 1995) (see the discussion in Section 4) points out that there are two perspectives on default reasoning. On the one hand there is the prototypical reading where $b \rightsquigarrow f$ is understood as "Birds typically fly." On the other hand, according to the presumptive reading it is read as "Birds are presumed to fly unless there is evidence to the contrary." The former was proposed in (Reiter et al., 1981) and Lehmann states that it is the intended reading for Rational Closure, whereas the presumptive reading is intended for the Lexicographic Closure. According to the prototypical reading the Drowning problem should not be solved. This is due to the fact 
that if there is an exception to some conditional with antecedent $A$ then the situation is not typical with respect to $A$. However, defaults with antecedent $A$ only account for typical situations (with respect to $A$ ). Hence, MP should not be applied to any conditional $A \rightsquigarrow B$ according to this view.

\subsection{Taking into account negative knowledge}

So far we focused on knowledge bases consisting on the one hand of conditionals and on the other hand of facts, i.e., facts expressed by propositions. It is interesting to enable the logic to also deal with knowledge bases including negative conditionals, i.e., formulas of the form $A \nLeftarrow \leftrightarrow B$. Note that the framework proposed in this paper is not able to deal with such knowledge bases in the case that our base system only consists of the core properties. Take for instance the simple penguin Example 2 and replace the premise $p \rightsquigarrow \neg f$ by $p \not \leftrightarrow f$. Note that for the logics $\mathbf{D P} \mathbf{p}^{\mathbf{x}}$ (where $\mathbf{x} \in\{\mathbf{r}, \mathbf{m}\}$ ) the unwanted $f$ is derivable for this premise set.

$\begin{array}{llllllll}1 & p \rightsquigarrow b & \text { PREM } & \emptyset & 4 & p & \text { PREM } & \emptyset \\ 2 & p \rightsquigarrow f & \text { PREM } & \emptyset & 5 & b & 1,4 ; \text { RU } & \{\bullet p\} \\ 3 & b \rightsquigarrow f & \text { PREM } & \emptyset & 6 & f & 3,5 ; \text { RU } & \{\bullet p, \bullet b\}\end{array}$

Note that there is no way to mark line 6 (in either of the strategies). However, as the following fact shows, the situation is different in case $\mathbf{R}$ is chosen as base system, i.e., for lower limit logic $\mathbf{R p}$.

Fact 3. The core properties, [RM], [rMP] and [Inh] imply ${ }^{23}$

$$
\begin{aligned}
\vdash(A \wedge(A \rightsquigarrow B \rightsquigarrow C) \wedge(A \not \hookrightarrow C)) \supset \bullet B \quad \text { [Spe'] } \\
\vdash\left(A \wedge\left(A \rightsquigarrow B_{1} \rightsquigarrow \ldots \rightsquigarrow B_{n} \rightsquigarrow C\right) \wedge(A \not C)\right) \supset \bullet B_{n} \quad[\text { [SpeG'] } \\
\vdash\left(A \wedge\left(A \rightsquigarrow B_{1} \rightsquigarrow \ldots \rightsquigarrow B_{n}\right) \wedge\left(B_{n} \rightsquigarrow D\right) \wedge\right. \\
\left.\left(A \rightsquigarrow C_{1} \rightsquigarrow \ldots \rightsquigarrow C_{m} \rightsquigarrow D\right) \wedge\left(B_{n} \rightsquigarrow \ldots \rightsquigarrow C_{m}\right)\right) \supset \bullet C_{m} \quad[\text { [PreE'] }
\end{aligned}
$$

In $\mathbf{D R} \mathbf{p}^{\mathbf{x}} f$ is not derivable since line 6 is marked by the following extension of the proof:

$$
7 \bullet b \quad 1,2,3,4 ; \text { Spe' } \emptyset
$$

\section{Conclusion}

In this paper an adaptive logic approach to Modus Ponens for conditional logics of normality was presented. By adaptively enhancing a given base logic we enrich it by the ability to model actual default inferencing. By means of benchmark examples it was demonstrated that the adaptive systems deal with specificity and conflicting

23. The proofs can be found in the Appendix. 
arguments in an intuitive way. The two adaptive standard strategies have been shown to correspond to two different intuitions: a more skeptical and a more credulous one.

\section{Acknowledgements}

Research for this paper was supported by the Research Fund of Ghent University by means of Research Project 01G01907. I thank Joke Meheus and Dunja Šešelja and the three anonymous reviewers for valuable comments to a former version of this paper.

\section{APPENDIX}

\section{A. Some proofs}

Lemma 1. The core properties, [rMP] and [Inh] entail $\left(A_{1} \wedge\left(A_{1} \rightsquigarrow \ldots \rightsquigarrow A_{n} \rightsquigarrow B\right)\right) \supset$ $\left(B \vee \bullet A_{n}\right)$.

Proof. Due to $A_{1}, A_{1} \rightsquigarrow A_{2}$ and [rMP] we have $A_{2} \vee \bullet A_{1}$. Analogously we get $A_{3} \vee \bullet A_{2} \vee \bullet A_{1}$ and finally $B \vee \bullet A_{n} \vee \cdots \vee \bullet A_{1}$. By iterated applications of [Inh] we get $B \vee \bullet A_{n}$.

Proof of Fact 1. By means of $A, A \rightsquigarrow B \rightsquigarrow C$ and the lemma, (†) $C \vee \bullet B$. By means of $A$ and $A \rightsquigarrow \neg C$, by (rMP) $\neg C \vee \bullet A$. Since $A \rightsquigarrow B$, by [Inh], $\bullet A \supset B$. Hence, $(\ddagger) \neg C \vee \bullet B$. By $(\dagger)$ and $(\ddagger), \bullet$.

Proof of Fact 2. "[Spe1]": this is trivial. "[SpeG]": By Lemma 1, $C \vee \bullet B_{n}$ and $\neg C \vee \bullet A$. By multiple applications of [Inh], $\bullet A \supset B_{n}$. Hence $\neg C \vee \bullet B_{n}$. Thus, $\bullet B_{n}$. [Spe2] and [sSpe] follow immediately with [SpeG] and [CI]. "[PreE]": By Lemma $1, D \vee \bullet B_{n}$ and $\neg D \vee \bullet C_{m}$. By multiple applications of [Inh], $\bullet B_{n} \supset \bullet C_{m}$. Hence, $D \vee \bullet C_{m}$. Thus, $\bullet C_{m}$. "[Conf]": By Lemma $1, D_{i} \vee \bullet B_{i}$. Due to $\neg \bigwedge_{I} D_{i}$ and by classical logic, $\bigvee_{I} \bullet B_{i}$.

Proof of Fact 3. "[Spe']": Suppose $\neg \bullet B$. Then, due to [Spe1], $B \not \neg \neg A$. By (RM), $(A \wedge B) \rightsquigarrow$ $C$. But then by [RT], $A \rightsquigarrow C$,-a contradiction. "[SpeG']": Suppose $\neg \bullet B_{n}$. Hence, due to [Inh], $\neg \bullet B_{i}$ for all $i<n$ and $\neg \bullet A$. Hence, due to [Spe'], $A \rightsquigarrow B_{i}$ for all $i \leq n$ (otherwise, $\bullet B_{i}$ ). But then by [Spe'], $\bullet B_{n},-$ a contradiction. "[PreE']": similar and left to the reader.

\section{B. The semantics}

I focused in this paper on the base logics $\mathbf{L} \in\{\mathbf{P}, \mathbf{R}\}$. There are many semantics around for the core properties (see Footnote 2). Paradigmatically I will extend the semantics based on preferential models (see (Kraus et al., 1990)) for our lower limit logics Lp. Again there are various ways to enhance preferential models such as to serve as semantical representations of Lp. I am going to present versions which are technically straightforward. In this appendix I 
will cover the case for $\mathbf{L}=\mathbf{P}$ and hence the logic $\mathbf{P} \mathbf{p}$. However, for $\mathbf{R} \mathbf{p}$ the semantics are defined analogously.

We call interpretations $\mathcal{W} \rightarrow\{0,1\}$ which satisfy the classical truth conditions for $\wedge, \vee, \neg, \supset$ and $\equiv$ classical propositional worlds and write $\mathcal{U}$ for the set of all classical propositional worlds.

Definition 6. Let $\prec$ be a partial order on a set $U$ and $V \subseteq U$. We say that $x \in V$ is minimal in $V$ iff there is no $y \in V$, such that $y \prec x$. We shall say that $V$ is smooth iff for all $x \in V$, either there is a $y$ minimal in $V$, such that $y \prec x$ or $x$ is itself minimal.

Definition 7. A preferential model $M$ is a triple $\langle S, l, \prec\rangle$ where $S$ is a set, the elements of which will be called states, $l: S \rightarrow \mathcal{U}$ assigns a classical propositional world to each state and $\prec$ is a strict partial order on $S$ satisfying the following smoothness condition: for all $A \in \mathcal{W}$, the set of states $\hat{A}={ }_{\text {df }}\{s \mid s \in S, s \equiv A\}$ is smooth, where $\equiv$ is defined as $s \equiv A$ (read $s$ satisfies $A$ ) iff $l(s)(A)=1$. $M$ validates $A \rightsquigarrow B$, in signs $M \models A \rightsquigarrow B$, iff, for any $s$ minimal in $\hat{A}, s \equiv B$. For the classical connectives $\models$ is defined as usual:

$$
\begin{array}{r}
M \models A \vee B \text { iff } M \models A \text { or } M \models B \\
M \models A \wedge B \text { iff } M \models A \text { and } M \models B \\
M \models \neg A \text { iff } M \not \models A \\
M \models A \supset B \text { iff } M \models \neg A \vee B \\
M \models A \equiv B \text { iff } M \models A \supset B \text { and } M \models B \supset A
\end{array}
$$

where $A$ and $B$ are in the $(\wedge, \vee, \supset, \neg, \equiv)$-closure of $\mathcal{W}^{\rightsquigarrow}$ and $\mathcal{W}^{\rightsquigarrow}$ is the set of all conditionals.

Let $\mathcal{W}^{\bullet}$ be the set of all formulas of the form $\bullet A$. Let $\mathcal{P}$ be the $(\wedge, \vee, \supset, \neg, \equiv)$-closure of $\mathcal{W} \cup \mathcal{W}^{\bullet} \cup \mathcal{W}^{\rightsquigarrow}$. We have two tasks in order to define the semantics for Pp. On the one hand, preferential models have to be generalized in order to allow for the modeling of factual premises. On the other hand, the new rules [rMP] and [Inh] have to be taken into account. We will realize both requirements by introducing an actual world to the preferential models defined above.

Definition 8. A preferential $l_{c}$ model $M$ with an actual world is defined by $\langle S, l, \prec, @\rangle$ where $M^{\prime}=\langle S, l, \prec\rangle$ is a preferential model and @ is an interpretation $\mathcal{P} \rightarrow\{0,1\}$ such that the classical clauses (where now $A, B \in \mathcal{P}$ ) $[\mathrm{S}-\vee],[\mathrm{S}-\wedge],[\mathrm{S}-\neg],[\mathrm{S}-\supset]$, and $[\mathrm{S}-\equiv]$ and the following rules are valid:

$$
\begin{array}{r}
M^{\prime} \models A \rightsquigarrow B \text { iff } @(A \rightsquigarrow B)=1 \\
\text { If } @(A)=@(A \rightsquigarrow B)=1 \text { and } @(\bullet A)=0, \text { then } @(B)=1 \\
\text { If } @(\bullet A)=@(A \rightsquigarrow B)=1, \text { then } @(\bullet B)=1
\end{array}
$$

We define $M \models \varphi$ iff $@(\varphi)=1$. We denote the corresponding semantic consequence relation by $\Vdash_{\mathbf{P}}^{\mathbf{P}}$ which is defined in the usual way: $\Gamma \Vdash_{\mathbf{P}}^{\mathbf{P}} \varphi$ iff all preferential ${ }_{c}$ models $M$ with an actual world that verify all members of $\Gamma$ also verify $\varphi$.

Lemma 2. Let $\Gamma \subset \mathcal{P}$ be a $\mathbf{P} \mathbf{p}$-consistent premise set. There is a preferential model $M$ with an actual world for which $M \models \Gamma$. 
Sketch of the proof. Let $\Gamma^{\prime}$ be a maximal consistent (w.r.t. Pp) extension of $\Gamma$. Take any preferential model $M^{\prime}$ of $\Gamma^{\prime} \cap \mathcal{W}^{\rightsquigarrow}$. Obviously such a model exists since $\Gamma^{\prime} \cap \mathcal{W}^{\rightsquigarrow}$ is $\mathbf{P p}$-consistent. Let @ be defined by @ $(A)=1$ iff $A \in \Gamma^{\prime}$. Let $M=\left\langle M^{\prime}\right.$, @ $\rangle$. Obviously @ fulfills the rules [S-@], [S-rMP], [S-Inh], the classical rules and the core properties.

Theorem 1. If $\Gamma \vdash_{\mathbf{P p}} \varphi$ then $\Gamma \vdash_{\mathbf{P}}^{\mathbf{p}} \varphi$.

Sketch of the proof. The proof proceeds via an induction over the derivative steps constituting a proof of $\varphi$.

" $n=1$ ": If $\varphi$ is derived by a core rule $\mathcal{R}$, then the antecedents of the rule are valid in all models $M=\left\langle M^{\prime}, @\right\rangle$ of $\Gamma$ since they are in $\Gamma$ and due to the fact that $M^{\prime}$ is a preferential model, $\varphi$ is also valid in $M^{\prime}$. By [S-@], $\varphi$ is valid in $M$. If $\varphi=B$ has been derived by [rMP] from $A, A \rightsquigarrow B$, and $\neg \bullet A$, then $A, A \rightsquigarrow B, \neg \bullet A \in \Gamma$. By [S-rMP] and [S-@], $B$ is valid in all models. For [S-Inh] and the classical rules the argument is similar.

" $n \rightarrow n+1$ ": Let $\varphi$ be derived by a core rule $\mathcal{R}$. All antecedents of the rule are valid in all models $M=\left\langle M^{\prime}, @\right\rangle$ of $\Gamma$ and since $M^{\prime}$ is a preferential model, also the consequent of $\mathcal{R}$ is valid in $M^{\prime}$. By [S-@], $\varphi$ is valid in $M$. If $\varphi=B$ has been derived by [rMP] from $A, A \rightsquigarrow B$, and $\neg \bullet A$, then $\Gamma \Vdash_{\mathbf{P}}^{\mathbf{p}} A, A \rightsquigarrow B, \neg \bullet A$. By [S-rMP], $B$ is valid in all models. For [S-Inh] and the classical rules the argument is similar.

Theorem 2. If $\Gamma \Vdash_{\mathbf{P}}^{\mathbf{p}} \varphi$ then $\Gamma \vdash_{\mathbf{P p}} \varphi$.

Proof. Suppose $\Gamma \nvdash \mathbf{P p} \varphi$, then $\Gamma \cup\{\neg \varphi\}$ is Pp-consistent. Thus, by Lemma 2, there is a preferential $_{c}$ model with an actual world for $\Gamma \cup\{\neg \varphi\}$.

So far I have presented the semantics for the adaptive logics based on the core properties, i.e., based on $\mathbf{P}$. For $\mathbf{R} \mathbf{p}$ the semantics are defined analogously. Instead of preferential models, ranked models are used. Ranked models are preferential models for which $\prec$ is modular (see (Lehmann et al., 1992) for details). The completeness and soundness results are shown analogously. The easy meta-proofs are left to the reader.

\section{References}

Adams E. W., The Logic of Conditionals, D. Reidel Publishing Co., 1975.

Arieli O., Avron A., " General patterns for nonmonotonic reasoning: from basic entailments to plausible relations", Logic Journal of the IGPL, vol. 8, n² 2, p. 119-148, March, 2000.

Batens D., “ A Universal Logic Approach to Adaptive Logics”, Logica Universalis, vol. 1, p. 221-242, 2007.

Batens D., Straßer C., Verdée P., “ On the Transparency of Defeasible Logics: Equivalent Premise Sets, Equivalence of Their Extensions, and Maximality of the Lower Limit", Logique et Analyse, vol. 52, n 207, p. 281-304, 2009.

Benferhat S., Cayrol C., Dubois D., Lang J., Prade H., " Inconsistency management and prioritized syntax-based entailment", IJCAI'93: Proceedings of the 13th international joint conference on Artifical intelligence, Morgan Kaufmann Publishers Inc., San Francisco, CA, USA, p. 640-645, 1993. 
Bonevac D., Deduction, Introductory Symbolic Logic, 2nd ed. edn, Blackwell Publishers, 2003.

Booth R., “ The lexicographic closure as a revision process”, Journal of Applied Non-Classical Logics, vol. 11, n 1-2, p. 35-58, 2001.

Boutilier C., "Conditional logics of normality: a modal approach", Artificial Intelligence, vol. $68, n^{\circ} 1$, p. 87-154, 1994a.

Boutilier C., " Unifying default reasoning and belief revision in a modal framework", Artificial Intelligence, vol. 68, $\mathrm{n}^{\circ}$ 1, p. 33-85, 1994b.

Broda K., Gabbay D. M., Lamb L. C., Russo A. M., " Labelled Natural Deduction for Conditional Logics of Normality", Logic Journal of the IGPL, vol. 10, n², p. 123-163, March, 2002.

Chellas B. F., “ Basic Conditional Logic”, Journal of Philosophical Logic, vol. 4, n² 2, p. 133 $153,1975$.

de Kleer J., “ An assumption-based TMS”, Readings in nonmonotonic reasoning, Morgan Kaufmann Publishers Inc., San Francisco, CA, USA, p. 280-298, 1987.

Delgrande J., “ An approach to default reasoning based on a first-order conditional logic: revised report”, Artificial Intelligence, vol. 36, n 1, p. 63-90, 1988.

Delgrande J., “ On a rule-based interpretation of default conditionals”, Annals of Mathematics and Artificial Intelligence, vol. 48, n 3-4, p. 135-167, December, 2006.

Dubois D., Prade H., “ Possibilistic logic, preferential models, non-monotonicity and related issues", In Proceedings Twelfth International Joint Conference on Artificial Intelligence, p. 419-424, 1991.

Dung P. M., Son T. C., “ An argument-based approach to reasoning with specificity”, Artificial Intelligence, vol. 133, $\mathrm{n}^{\circ}$ 1-2, p. 35-85, 2001.

Freund M., “ Default Extensions: Dealing with Computer Information”, Artificial Intelligence, vol. 92, n 1-2, p. 277-288, 1997.

Friedman N., Halpern J. Y., " Plausibility measures and default reasoning”, Journal of the ACM, vol. 48, p. 1297-1304, 1996.

Gärdenfors P., “Conditionals and Changes of Belief”, Acta Philosophica Fennica, 1978.

Garg D., Abadi M., “ A modal deconstruction of access control logics”, Proceedings of the Theory and practice of software, 11th international conference on Foundations of software science and computational structures, FOSSACS'08/ETAPS'08, Springer-Verlag, Berlin, Heidelberg, p. 216-230, 2008.

Geffner H., Pearl J., " Conditional entailment: bridging two approaches to default reasoning”, Artificial Intelligence, vol. 53, $\mathrm{n}^{\circ}$ 2-3, p. 209-244, 1992.

Genovese V., Giordano L., Gliozzi V., Pozzato G. L., “ A Conditional Constructive Logic for Access Control and Its Sequent Calculus.”, Proceedings of TABLEAUX'11, p. 164-179, 2011.

Ginsberg M., Essentials of Artificial Intelligence, Morgan Kaufmann Publishers Inc., San Francisco, CA, USA, 1994.

Giordano L., Gliozzi V., Olivetti N., Pozzato G., “ Analytic Tableau Calculi for KLM Rational Logic R", Proceedings of the 10th European Conference on Logics in Artificial Intelligence, ACM, New York, USA, p. 190-202, 2006.

Giordano L., Gliozzi V., Olivetti N., Pozzato G. L., “ A Nonmonotonic Extension of KLM Preferential Logic P.”, Objekttechnologie'10, p. 317-332, 2010a. 
Giordano L., Gliozzi V., Olivetti N., Pozzato G. L., “ Preferential vs Rational Description Logics: which one for Reasoning About Typicality?.”, ECAI'10, p. 1069-1070, $2010 \mathrm{~b}$.

Giordano L., Olivetti N., Gliozzi V., Pozzato G. L., “ALC + T: a Preferential Extension of Description Logics.”, Fundamenta Informaticaep. 341-372, 2009.

Goble L., “ A Proposal for Dealing with Deontic Dilemmas”, in A. Lomuscio, D. Nute (eds), DEON, vol. 3065 of Lecture Notes in Computer Science, Springer, p. 74-113, 2004.

Goldszmidt M., Pearl J., " On the relation between rational closure and system Z”, Third Int. Workshop Nonmonotonic Reasoning (South Lake Tahoe), p. 130-140, 1990.

Goldszmidt M., Pearl J., “Rank-based Systems: A Simple Approach to Belief Revision, Belief Update, and Reasoning about Evidence and Actions", Proceedings of KR'92, p. 661-672, 1992.

Goldzsmidt M., Morris P., Pearl J., “ A Maximum Entropy Approach to Nonmonotonic Reasoning”, IEEE Transactions on Pattern Analysis and Machine Intelligence, vol. 15, $\mathrm{n}^{\circ} 3$, p. 220-232, 1993.

Horty J. F., " Some direct theories of nonmonotonic inheritance", in D. Gabbay, C. J. Hogger, J. A. Robinson (eds), Handbook of Logic in Artificial Intelligence and Logic Programming, Volume 3: Nonmonotonic Reasoning and Uncertain Reasoning, Oxford University Press, p. 111-187, 1994.

Horty J. F., "Skepticism and floating conclusions", Artificial Intelligencep. 55-72, February, 2002.

Jeffry P. F., Renee E., “ On Relevance in Nonmonotonic Reasoning: Some Empirical Studies”, in G. Russ, S. Devika (eds), Relevance: AAAI 1994 Fall Symposium Series, Palo Alto: AAAI Press, p. 64-67, 1994.

Koons R., “Defeasible Reasoning”, in E. N. Zalta (ed.), The Stanford Encyclopedia of Philosophy, winter 2009 edn, 2009.

Kraus S., Lehmann D. J., Magidor M., “ Nonmonotonic Reasoning, Preferential Models and Cumulative Logics”, Artificial Intelligence, vol. 44, p. 167-207, 1990.

Lamarre P., “S4 as the Conditional Logic of Nonmonotonicity”, Proceedings of KR'91, p. 357367, 1991.

Lamarre P., A family of Non-Monotonic Inference Systems based on Conditional Logics, Technical report, AAAI FS-93-01, 1993.

Lehmann D. J., “ Another Perspective on Default Reasoning”, Annals of Mathematics and Artificial Intelligence, vol. 15, n 1, p. 61-82, 1995.

Lehmann D. J., Magidor M., “What does a conditional knowledge base entail?”, Artificial Intelligence, vol. 55, $\mathrm{n}^{\circ}$ 1, p. 1-60, 1992.

Lewis D., Counterfactuals, Blackwell Publishers, December, 2000.

Makinson D., Schlechta K., "Floating conclusions and zombie paths: two deep difficulties in the "directly skeptical" approach to defeasible inheritance nets", Artificial Intelligence, vol. 48, n'2, p. 199-209, 1991.

McCarthy J., " Circumscription - A Form of Non-Monotonic Reasoning”, Artificial Intelligence, vol. 13, p. 27-29, 1980.

Moore R. C., “ Possible-World Semantics for Autoepistemic Logic.”, Proceedings of AAAI non-monotonic reasoning Workshop, p. 344-354, 1984. 
Neufeld E., "Defaults and probabilities: extensions and coherence", Proceedings of the first international conference on Principles of knowledge representation and reasoning, Morgan Kaufmann Publishers Inc., San Francisco, CA, USA, p. 312-323, 1989.

Nute D., Topics in conditional logic, Philosophical studies series in philosophy, 20, D. Reidel Pub. Co., 1980.

Pearl J., "Probabilistic semantics for nonmonotonic reasoning: a survey", Proceedings of the first international conference on Principles of knowledge representation and reasoning, Morgan Kaufmann Publishers Inc., San Francisco, CA, USA, p. 505-516, 1989.

Pearl J., " System Z: a natural ordering of defaults with tractable applications to nonmonotonic reasoning", TARK '90: Proceedings of the 3rd conference on Theoretical aspects of reasoning about knowledge, Morgan Kaufmann Publishers Inc., San Francisco, CA, USA, p. 121-135, 1990.

Pollock J., “ Defeasible Reasoning”, Cognitive Science, vol. 11, n 4, p. 481-518, 1987.

Poole D., " The effect of knowledge on belief: conditioning, specificity and the lottery paradox in default reasoning”, Artificial Intelligence, vol. 49, n 1-3, p. 281-307, 1991.

Putte F. V. D., Straßer C., " Extending the Standard Format of Adaptive Logics to the Prioritized Case", Logique et Analyse (Forthcoming), 201X. Forthcoming.

Reiter R., “A Logic for Default Reasoning”, Artificial Intelligence, 1980.

Reiter R., Criscuolo G., “ On interacting defaults”, IJCAI'81: Proceedings of the 7th international joint conference on Artificial intelligence, Morgan Kaufmann Publishers Inc., San Francisco, CA, USA, p. 270-276, 1981.

Schröder L., Pattinson D., Hausmann D., “ Optimal Tableaux for Conditional Logics with Cautious Monotonicity", Proceeding of the 2010 conference on ECAI 2010: 19th European Conference on Artificial Intelligence, IOS Press, Amsterdam, The Netherlands, The Netherlands, p. 707-712, 2010.

Shoham Y., “ A Semantical Approach to Nonmonotonic Logics”, in M. L. Ginsberg (ed.), Readings in Non-Monotonic Reasoning, Morgan Kaufmann, Los Altos, CA, p. 227-249, 1987.

Shoham Y., Reasoning about Change: Time and Causation from the Standpoint of Artificial Intelligence, M.I.T. Press, Cambridge,Mass., 1988.

Spohn W., " Ordinal conditional functions: A dynamic theory of epistemic states", in W. L. Harper, B. Skyrms (eds), Causation in Decision, Belief Change and Statistics, Springer, p. 105-134, August, 1988.

Stalnaker R. F., “A Theory of Conditionals”, in N. Reischer (ed.), Studies in Logical Theory, Basil Blackwell, 1968.

Stalnaker R. F., “What is a Nonmonotonic Consequence Relation?”, Fundamenta Informaticae, vol. 21, n 1/2, p. 7-21, 1994.

Straßer C., “ An adaptive logic for Rational Closure”, in W. Carnielli, M. E. Coniglio, I. M. L. D’Ottaviano (eds), The Many Sides of Logic, College Publications, p. 47-67, 2009.

Straßer C., " A deontic logic framework allowing for factual detachment”, Journal of Applied Logic, vol. 9, n 1, p. 61-80, 2010.

Wobcke W., “Belief Revision, Conditional Logic and Nonmonotonic Reasoning”, Notre Dame Journal of Formal Logic, vol. 36, n 1, p. 55-102, 1995. 